\title{
РОЗРАХУНОК КРИТИЧНОЇ ТЕМПЕРАТУРИ БОЗЕ-ГАЗУ $З$ ДАЛЕКОДІЙНИМИ СИЛАМИ
}

\author{
В.С. ПАСТУХОВ
}

УДК 538.94
(C) 2012

Львівський національний університет ім. Івана Франка

(Вул. Драгоманова, 12, Лъвів, 79005; e-mail: volodyapastukhov@gmail. com)

У статті обчислено критичну температуру моделі бозе-газу зі степеневим законом залежності потенціальної енергії попарної взаємодії від відстані між частинками. У граничних випадках результат відтворює відомі результати з літератури. У наближенні хаотичних фаз (RPA) проаналізовано параметри спектра колективних збуджень моделі та отримано довгохвильову асимптотику структурного фактора системи при температурах, вищих за температуру фазового переходу.

\section{1. Вступ}

Історія вивчення бозе-систем з далекодійними силами почалась з роботи Фолді [1] - першої в теорії зарядженого бозе-газу, зануреного в компенсуюче поле, де в наближенні Боголюбова досліджували властивості основного стану системи. Незначна кількість робіт [2-5], в яких розглядали критичну поведінку моделі, з'явилася лише в першій половині 70-х pp. ХХ ст. разом з бурхливим розвитком теорії фазових переходів і критичних явищ. В основному автори обмежувались самоузгодженим, статичним варіантом RPAнаближення, яке дає змогу знайти головні внески у термодинамічні функції системи в околі температури фазового переходу та обчислити критичні індекси моделі.

У цій статті поставлено за мету розрахунок критичної температури моделі бозе-системи зі степеневим законом поведінки потенціалу парної взаємодії між частинками вигляду $1 / r^{1+\sigma}$, принаймні на великих відстанях. Якісно основний стан цієї моделі для довільної вимірності простору $d$ у границі $\sigma=1$ проаналізовано за допомогою ренормгрупових методів у роботі [6]. У цікавому випадку $d=1$ отримано повну узгодженість з точним розв'язком. Критичну поведінку нашої моделі не досліджували.

Отже, ми розглядаємо сукупність $N$ безспінових частинок в об'ємі $V$ тривимірного простору при температурі $T$. Зручніше працювати у великому канонічному ансамблі, тому введемо в розгляд хімічний потенціал $\mu$. Фур'є-образ потенціалу попарної взаємодії виберемо в такому вигляді $\left(\lambda_{\sigma}>0\right)$ :

$\nu_{\sigma}(k)=\frac{\lambda_{\sigma}}{k^{2-\sigma}}, \quad 0 \leq \sigma \leq 2$.

Повністю аналогічно до моделі зарядженого бозе-газу в компенсуючому полі, де стійкість системи в термодинамічній границі $(N \rightarrow \infty, V \rightarrow \infty$, але густина $\rho=N / V=$ const) забезпечується однорідним фоном з протилежним за знаком зарядом так, щоб у цілому система була електронейтральною, накладемо на нульову компоненту фур'є-образу потенціалу умову $\nu_{\sigma}(0)=0(\sigma \neq 2)$. Окремо потрібно розглядати випадок $\sigma=2\left(\lambda_{2}=4 \pi \hbar^{2} a / m\right.$, де $a-$ довжина $s$-розсіяння), який відповідає моделі $3 \delta$ відштовхуванням. Явний вигляд потенціалу парної взаємодії між частинками:

$\Phi_{\sigma}(r)=\frac{1}{2^{2-\sigma} \pi^{3 / 2}} \frac{\Gamma(1 / 2+\sigma / 2)}{\Gamma(1-\sigma / 2)} \frac{\lambda_{\sigma}}{r^{1+\sigma}}, \quad \sigma \leq 2$,

де $\Gamma(x)$ - гамма-функція. Для нашої моделі можна сконструювати три параметри з розмірністю оберненої довжини. Перший - найбільш простий, пов'язаний $з$ рівноважною густиною системи і пропорційний до $\rho^{1 / 3}$, другий - характерний для ідеального бозе-газу при скінченних температурах $k_{0}=$ $p_{0}=\sqrt{2 m T} / \hbar$, і нарешті, третій $k_{\sigma}=\left(\rho \lambda_{\sigma} / T\right)^{1 /(2-\sigma)}$ $(\sigma \neq 2)$, який у випадку кулонівського потенціалу $\left(\lambda_{0}=4 \pi e^{2}\right)$ переходить в обернений радіус Дебая $k_{\sigma=0} \equiv k_{D}=\sqrt{4 \pi e^{2} \rho / T}$. 3 цих величин можна сконструювати два знерозмірених параметри. При скінченних температурах теорія збурень будується за відношенням $k_{\sigma} / k_{0}$, яке в околі температури бозе-конденсації $T_{0}$ ідеального газу пропорційне до $\left[\lambda_{\sigma} m \rho^{(\sigma-1) / 3} / \hbar^{2}\right]^{1 /(2-\sigma)}$. У крайньому граничному випадку кулонівського газу, $\sigma=0$, цей розклад еквівалентний розкладу за так званим параметром Бракнеpa $r_{s}=(3 / 4 \pi \rho)^{1 / 3} \frac{m e^{2}}{\hbar^{2}}-$ відношення середньої відстані між частинками до борівського радіуса. У протилежній границі $\sigma=2$ потрібно замінити $k_{\sigma}^{2-\sigma} \rightarrow \rho \lambda_{2} / T$, а розклад ряду теорії збурень в околі $T_{c}$ ведеться за газовим параметром $a \rho^{1 / 3}$. 
Основним станом моделі для вимірностей простору, більше двох, є стан з розмитим взаємодією бозеконденсатом. Для опису його властивостей при малих значеннях параметра неідеальності достатньо використати метод наближеного вторинного квантування Боголюбова. Зрозуміло, що для вимірності простору $d=3$ у граничному випадку бозе-системи з кулонівською взаємодією відтворюються результати роботи [1], а для моделі слабонеідеального бозе-газу отримуються відомі результати Лі-Янга [7]. Довгохвильова асимптотика спектра елементарних збуджень над основним станом має характерну поведінку: $E_{\sigma}(k) \sim$ $k^{\sigma / 2}$.

\section{2. Структурний фактор та спектр колективних збуджень}

Спочатку проаналізуємо структурний фактор моделі:

$S_{\sigma}(k)=\int_{-\infty}^{\infty} d \omega S_{\sigma}(\omega, k)$

де введено позначення для динамічного структурного фактора в наближенні хаотичних фаз $(\beta=1 / T)$ :

$$
\begin{aligned}
& S_{\sigma}(\omega, k)=\frac{1}{\pi \rho} \frac{1}{1-e^{-\beta \omega}} \times \\
& \times \frac{I(\omega, k)}{\left(1+\nu_{\sigma}(k) R(\omega, k)\right)^{2}+\left(\nu_{\sigma}(k) I(\omega, k)\right)^{2}} .
\end{aligned}
$$

Тут $R(\omega, k), I(\omega, k)$ (див. додаток) - дійсна та уявна частини поляризаційного оператора $\left(n(x)=\left\{e^{x}-\right.\right.$ $\left.1\}^{-1}\right)$

$$
\begin{aligned}
& \Pi\left(\omega_{n}, k\right)=\frac{1}{V} \sum_{\mathbf{p}} n\left(\beta \xi_{p}\right) \times \\
& \times\left\{\frac{1}{\xi_{|\mathbf{p}-\mathbf{k}|}-\xi_{p}-i \omega_{n}}+\left(\omega_{n} \rightarrow-\omega_{n}\right)\right\},
\end{aligned}
$$

після аналітичного продовження $i \omega_{n} \rightarrow \omega+i 0$. Тут введено позначення $\xi_{p}=\varepsilon_{p}-\mu$, де $\varepsilon_{p}=\hbar^{2} p^{2} / 2 m$ - вільночастинковий спектр, $\omega_{n}=2 \pi n T, \quad(n=$ $0, \pm 1, \ldots)$ - матсубарівська частота. Враховуючи аргументацію [8], запишемо структурний фактор в довгохвильовій області $\left(k \ll k_{\sigma}\right)$ :

$$
S_{\sigma}(k)=\frac{1}{\rho \nu_{\sigma}^{2}(k)}[\partial R(\omega, k) / \partial \omega]_{\omega \rightarrow \omega_{\sigma}(k)}^{-1} \times
$$

$\times \operatorname{coth}\left(\beta \omega_{\sigma}(k) / 2\right)$,

де $\omega_{\sigma}(k)$ - спектр колективних збуджень системи, який визначається рівнянням

$1+\nu_{\sigma}(k) R\left(\omega_{\sigma}(k), k\right)=0$.

Формально диференціюючи це рівняння за $\nu_{\sigma}(k)$, отримуємо корисне співвідношення

$[\partial R(\omega, k) / \partial \omega]_{\omega \rightarrow \omega_{\sigma}(k)}^{-1}=\nu_{\sigma}^{2}(k) \frac{\partial \omega_{\sigma}(k)}{\partial \nu_{\sigma}(k)}$.

Перший момент динамічного структурного фактора повинен задовольняти умову

$$
\int_{-\infty}^{\infty} d \omega \omega S_{\sigma}(\omega, k)=\frac{\hbar^{2} k^{2}}{2 m}
$$

що в нашому випадку у довгохвильовій області приводить до співвідношення

$\frac{1}{\rho \nu_{\sigma}^{2}(k)}[\partial R(\omega, k) / \partial \omega]_{\omega \rightarrow \omega_{\sigma}(k)}^{-1}=\frac{\varepsilon_{k}}{\omega_{\sigma}(k)}$,

а з врахуванням рівності (6) - до залежності

$\omega_{\sigma}^{2}(k)=2 \rho \nu_{\sigma}(k) \varepsilon_{k}+\ldots$,

де трьома крапками позначено частину спектра, що не залежить від взаємодії. Зауважимо, що для малих значень взаємодії рівності (4)-(8) справедливі тільки в дуже вузькій області значень хвильового вектора в околі нуля. Спробуємо розширити область застосовності цих формул. Будемо вимагати, щоб формула (8) при вимкненні взаємодії, $\nu_{\sigma}(k) \rightarrow 0$, правильно відтворювала довгохвильову асимптотику структурного фактора ідеального бозе-газу. Зрозуміло, що при цьому ми повинні замінити реальний спектр колективних збуджень системи певним ефективним, для якого зберігаємо позначення $\omega_{\sigma}(k)$. Тоді для довгохвильової асимптотики "спектра" за відсутності взаємодії отримуємо

$\left.\omega_{\sigma}^{2}(k)\right|_{\nu_{\sigma}(k) \rightarrow 0}=2 T \varepsilon_{k} / S_{0}(k)$,

де $S_{0}(k)$ - структурний фактор ідеального бозе-газу. I нарешті, умова $S_{\sigma}(k \rightarrow \infty) \rightarrow 1$ однозначно визначає поведінку функції $\omega_{\sigma}(k \rightarrow \infty) \rightarrow \varepsilon_{k}$ при великих значеннях аргумента. Об'єднуючи все разом, отримуємо формулу для ефективного спектра системи

$\omega_{\sigma}^{2}(k)=2 \rho \nu_{\sigma}(k) \varepsilon_{k}+2 T \varepsilon_{k} / S_{0}(k)+\varepsilon_{k}^{2}$, 
яку слід підставити у вираз (4) для структурного фактора взаємодіючої системи

$S_{\sigma}(k)=\frac{\varepsilon_{k}}{\omega_{\sigma}(k)} \operatorname{coth}\left(\beta \omega_{\sigma}(k) / 2\right)$.

Цікаво, що тепер цей вираз правильно відтворює класичну границю теорї

$\left.S_{\sigma}(k)\right|_{\hbar \rightarrow 0}=\frac{1}{1+\beta \rho \nu_{\sigma}(k)}$.

Отже, ми отримали вираз, який коректно описує структурний фактор системи в довгохвильовій області $k \ll k_{0}$. Незважаючи на те, що співвідношення (10), (11) правильно відтворюють певні граничні випадки теорії, варто наголосити, що область застосування цих формул обмежена малими взаємодіями. Тобто високими густинами бозе-газу для $\sigma<1$ (низькими для $\sigma>1$ ). Поширення результатів на менші (більші) густини не є тривіальною задачею. 3 одного боку, це пов'язано з нашою процедурою "вгадування", 3 іншого боку, із застосовністю RPA-наближення.

Тепер досліджуватимемо спектр “плазмонів", тобто корені рівняння (5). Перепишемо це рівняння так (див. додаток):

$1-\left(\frac{k_{\sigma}}{k}\right)^{2-\sigma} \frac{k_{0}}{2 k}\left\{f\left(\beta E_{\sigma}(k) /\left(2 k / k_{0}\right)-k / 2 k_{0}, \beta \mu\right)-\right.$

$\left.-f\left(\beta E_{\sigma}(k) /\left(2 k / k_{0}\right)+k / 2 k_{0}, \beta \mu\right)\right\}=0$,

де для спектра введено нове позначення $E_{\sigma}(k)$. Поведінка спектра повністю визначається відношенням $k_{\sigma} / k_{0}$. По-перше, якщо $E_{\sigma}(k)-$ корінь рівняння, то й $-E_{\sigma}(k)$ теж. Враховуючи додатково визначеність функції $f(\varepsilon, y)$, переконуємось, що умова існування дійсних коренів рівняння (13) така:

$\frac{\beta E_{\sigma}(k)}{k / k_{0}}>\varepsilon_{0}(\beta \mu)$,

де $\varepsilon_{0}(y)$ - максимум функції $f(\varepsilon, y)$ по першій змінній при фіксованому значенні другої. По-друге, для всіх значень параметра $k_{\sigma} / k_{0}(\sigma<2)$ в області $k \ll k_{\sigma}$ існує одна гілка спектра, який у випадку зарядженого бозе-газу має характерну щілину $\omega_{0}=\sqrt{4 \pi e^{2} \hbar^{2} \rho / m}$. Щоб переконатись у цьому, достатньо використати розклад функції $f(\varepsilon, y)$ (див. додаток) при великих значеннях першого аргумента і підставити у рівняння (13). Тоді для спектра у границі $k_{\sigma} \gg k_{0}$ отримуємо

$E_{\sigma}^{2}(k)=2 \rho \nu_{\sigma}(k) \varepsilon_{k}+2 T \varepsilon_{k} \frac{g_{5 / 2}\left(e^{\beta \mu}\right)}{g_{3 / 2}\left(e^{\beta \mu}\right)}+\varepsilon_{k}^{2}$, що неважко переписати так [9]:

$E_{\sigma}^{2}(k)=2 \rho \nu_{\sigma}(k) \varepsilon_{k}+\frac{4}{3} \frac{K_{0}}{N} \varepsilon_{k}+\varepsilon_{k}^{2}$,

де $K_{0} / N$ - середня енергія ідеального бозе-газу з розрахунку на одну частинку.

Третьою важливою особливістю спектра колективних збуджень бозе-систем з нашим модельним потенціалом для $T>T_{c}$ є існування точки закінчення $k_{f}-$ найбільшого значення хвильового вектора, для якого рівняння (5) ще має корінь. На цю особливість спектра кулонівських систем не вказують автори статті [10], де досліджувалась довгохвильова асимптотика спектра плазмонів у зарядженому бозе-газі для всіх значень температури. У загальному випадку розміщення точки закінчення визначається параметром $k_{\sigma} / k_{0}$. Аналітично можна проаналізувати тільки граничні випадки малих і великих значень цього параметра. У випадку $k_{\sigma} / k_{0} \ll 1$, враховуючи рівняння $(13)$, легко бачити, що $E_{\sigma}\left(k_{f}\right) /\left(2 k_{f} / k_{0}\right)$ прямуватиме до $\varepsilon_{0}^{\prime}(\beta \mu)$ - точки мінімуму першої похідної функції $f^{\prime}(\varepsilon, \beta \mu)$ по змінній $\varepsilon$. Тоді для точки закінчення маEMO

$k_{f} / k_{\sigma}=\left(\left|f^{\prime}\left(\varepsilon_{0}^{\prime}(\beta \mu), \beta \mu\right)\right| / 2\right)^{1 /(2-\sigma)}, \quad k_{\sigma} \ll k_{0}$.

$\mathrm{У}$ протилежному випадку різниця $E_{\sigma}\left(k_{f}\right) /\left(2 k_{f} / k_{0}\right)-$ $k_{f} / 2 k_{0}$ прямує до точки максимуму $\varepsilon_{0}(\beta \mu)$ функції $f(\varepsilon, \beta \mu)$, а $E_{\sigma}\left(k_{f}\right) /\left(2 k_{f} / k_{0}\right)+k_{f} / 2 k_{0} \gg 1$, тоді відношення

$$
\frac{k_{f}}{k_{0}}=\left[\frac{1}{2}\left(\frac{k_{\sigma}}{k_{0}}\right)^{2-\sigma} f\left(\varepsilon_{0}(\beta \mu), \beta \mu\right)\right]^{1 /(3-\sigma)}, \quad k_{\sigma} \gg k_{0} .
$$

Нагадаємо, що ми працюємо у наближенні хаотичних фаз, в якому не зовсім коректно аналізувати границю $k_{\sigma} \gg k_{0}$.

Покажемо тепер, що загасання спектра

$\Gamma_{\sigma}(k)=I\left(E_{\sigma}(k), k\right)[\partial R(\omega, k) / \partial \omega]_{\omega \rightarrow E_{\sigma}(k)}^{-1}$

мале. Використовуючи формулу для функції $I(\omega, k)$ з додатка і останнє співвідношення, записуємо в довгохвильовій області

$\left.\frac{\Gamma_{\sigma}(k)}{E_{\sigma}(k)}\right|_{k \rightarrow 0}=\left(\frac{\pi}{8}\right)^{1 / 2}\left(\frac{k_{\sigma}}{k}\right)^{3(1-\sigma / 2)} \times$

$\times n\left(\frac{1}{2}\left(k_{\sigma} / k\right)^{2-\sigma}-\beta \mu\right) / g_{3 / 2}\left(e^{\beta \mu}\right)$, 
що в границі $\mu \rightarrow-\infty$ та $\sigma=0$ відтворює добре відомий результат

$\Gamma(k) / \omega_{0}=\left(\frac{\pi}{8}\right)^{1 / 2}\left(\frac{k_{D}}{k}\right)^{3} e^{-k_{D}^{2} / 2 k^{2}}$,

3 теорії класичної плазми.

Для випадку $\sigma=2$ поведінка спектра зовсім інша. У роботі [8] показано, що не для всіх значень параметра неідеальності $а \rho^{1 / 3}$ існують розв'язки рівняння (13), і тільки коли $4 \pi \hbar^{2} a \rho /(m T)>2 /\left|f^{\prime}\left(\varepsilon_{0}^{\prime}(\beta \mu), \beta \mu\right)\right|$ для $T>T_{c}$ виникають дві звукові гілки спектра, одна $з$ яких пригнічена значним загасанням. У критичній точці існує лише одна гілка спектра фононів для $4 \pi \hbar^{2} a \rho /\left(m T_{c}\right)>-\zeta(3 / 2) / \zeta(1 / 2)=1,789$.

\section{3. Одночастинкові стани}

Власноенергетична частина чи масовий оператор в RPA-наближенні [11]:

$\Sigma\left(\omega_{n}, p\right)=-\frac{1}{\beta V} \sum_{\omega_{n}^{\prime}} \sum_{\mathbf{k}} \frac{\nu_{\sigma}(k)}{1+\nu_{\sigma}(k) \Pi\left(\omega_{n}^{\prime}, k\right)} \times$

$\times \frac{1}{i\left(\omega_{n}+\omega_{n}^{\prime}\right)-\xi_{|\mathbf{p}+\mathbf{k}|}}$

де вже врахована рівність $\nu_{\sigma}(0)=0$. Після аналітичного продовження у верхню півплощину позначимо

$\Sigma_{R}(\omega, p)=\left.\operatorname{Re} \Sigma\left(\omega_{n}, p\right)\right|_{i \omega_{n} \rightarrow \omega+i 0}$,

$\Sigma_{I}(\omega, p)=\left.\operatorname{Im} \Sigma\left(\omega_{n}, p\right)\right|_{i \omega_{n} \rightarrow \omega+i 0}$.

Тепер можна записати рівняння для перенормованого спектра [12]:

$\widetilde{\xi}_{p}=\xi_{p}+\Sigma_{R}\left(\widetilde{\xi}_{p}, p\right)$.

Зрозуміло, що елементарні збудження будуть стійкими за умови, що загасання зникаюче мале:

$\gamma_{p}=Z(p) \Sigma_{I}\left(\widetilde{\xi}_{p}, p\right)$

$Z^{-1}(p)=1-\left.\frac{\partial \Sigma_{R}(\omega, p)}{\partial \omega}\right|_{\omega=\widetilde{\xi}_{p}}$

Рівноважна густина системи, а фактично рівняння для визначення хімічного потенціалу системи

$\rho=-\frac{1}{V} \sum_{\mathbf{p}} \lim _{\tau \rightarrow+0} \frac{1}{\beta} \sum_{\omega_{n}} \frac{e^{i \omega_{n} \tau}}{i \omega_{n}-\xi_{p}-\Sigma\left(\omega_{n}, p\right)}=$
$=\frac{1}{V} \sum_{\mathbf{p}} \int_{-\infty}^{\infty} \frac{d \omega}{\pi} \frac{n(\beta \omega) \Sigma_{I}(\omega, p)}{\left(\omega-\xi_{p}-\Sigma_{R}(\omega, p)\right)^{2}+\Sigma_{I}^{2}(\omega, p)}$

можна переписати так:

$\rho=\frac{1}{V} \sum_{\mathbf{p}} Z(p) n\left(\beta \widetilde{\xi}_{p}\right)$

3 якого ми знайдемо перенормовану температуру бозе-конденсації за умови $\widetilde{\mu}=0$. Далі розрахунки для масового оператора виконано тільки для температур в околі критичної, строго кажучи, у границі $T \rightarrow T_{c}+0$ та ідейно близькі до розрахунків роботи [13]. Виділяємо з формули (21) хартрі-фоківський внесок

$\Sigma\left(\omega_{n}, p\right)=\frac{1}{V} \sum_{\mathbf{k}} \nu_{\sigma}(k) n\left(\beta \xi_{|\mathbf{k}-\mathbf{p}|}\right)+$

$+\frac{1}{\beta V} \sum_{\omega_{n}^{\prime}} \sum_{\mathbf{k}} \nu_{\sigma}(k) \frac{\nu_{\sigma}(k) \Pi\left(\omega_{n}^{\prime}, k\right)}{1+\nu_{\sigma}(k) \Pi\left(\omega_{n}^{\prime}, k\right)} \times$

$\times \frac{1}{i\left(\omega_{n}+\omega_{n}^{\prime}\right)-\xi_{|\mathbf{p}+\mathbf{k}|}}$

і дріб у другій сумі переписуємо, використовуючи спектральні співвідношення

$\frac{\nu_{\sigma}(k) \Pi\left(\omega_{n}^{\prime}, k\right)}{1+\nu_{\sigma}(k) \Pi\left(\omega_{n}^{\prime}, k\right)}=\frac{1}{\pi} \int_{-\infty}^{\infty} \frac{d \omega}{\omega-i \omega_{n}^{\prime}} \times$

$\times \frac{\nu_{\sigma}(k) I(\omega, k)}{\left(1+\nu_{\sigma}(k) R(\omega, k)\right)^{2}+\left(\nu_{\sigma}(k) I(\omega, k)\right)^{2}}$,

після цього елементарно беремо суму за $\omega_{n}^{\prime}$ :

$\Sigma\left(\omega_{n}, p\right)=\frac{1}{V} \sum_{\mathbf{k}} \nu_{\sigma}(k) n\left(\beta \xi_{|\mathbf{k}-\mathbf{p}|}\right)+$

$+\frac{1}{V} \sum_{\mathbf{k}} \nu_{\sigma}(k) \int_{-\infty}^{\infty} \frac{d \omega}{\pi} \frac{n(\beta \omega)-n\left(\beta \xi_{|\mathbf{k}-\mathbf{p}|}\right)}{\omega-\xi_{|\mathbf{k}-\mathbf{p}|}+i \omega_{n}} \times$

$\times \frac{\nu_{\sigma}(k) I(\omega, k)}{\left(1+\nu_{\sigma}(k) R(\omega, k)\right)^{2}+\left(\nu_{\sigma}(k) I(\omega, k)\right)^{2}}$.

Зрозуміло, враховуючи складність виразу, що для проведення подальших розрахунків потрібно застосувати чергове спрощення. Оскільки в цій області є 
добре визначена гілка спектра колективних збуджень системи для значень хвильового вектора, менших від $k_{f}$, то вираз (30) переписуємо, виділяючи в сумах за хвильовим вектором $\mathbf{k}$ значення менші та більші $k_{f}$. Тоді під сумами за $k \leq k_{f}$ інтеграли за змінною $\omega$ можна наближено розрахувати, використавши співвідношення

$$
\begin{aligned}
& \frac{1}{\pi \rho} \frac{I(\omega, k)}{\left(1+\nu_{\sigma}(k) R(\omega, k)\right)^{2}+\left(\nu_{\sigma}(k) I(\omega, k)\right)^{2}} \rightarrow \\
& \rightarrow \frac{1}{\rho \nu_{\sigma}(k)} \operatorname{sign}(I(\omega, k)) \delta\left(1+\nu_{\sigma}(k) R(\omega, k)\right),
\end{aligned}
$$

враховуючи малість загасання спектра колективних збуджень системи. Остаточно маємо

$$
\Sigma\left(\omega_{n}, p\right)=\frac{1}{V} \sum_{\mathbf{k}, k \leq k_{f}} \nu_{\sigma}(k) n\left(\beta \xi_{|\mathbf{k}-\mathbf{p}|}\right)-
$$

$-\frac{1}{V} \sum_{\mathbf{k}, k \leq k_{f}}\left[\partial R\left(E_{\sigma}(k), k\right) / \partial E_{\sigma}(k)\right]^{-1} \times$

$$
\begin{aligned}
& \times\left\{\frac{1+n\left(\beta E_{\sigma}(k)\right)+n\left(\beta \xi_{|\mathbf{k}-\mathbf{p}|}\right)}{E_{\sigma}(k)+\xi_{|\mathbf{k}-\mathbf{p}|}-i \omega_{n}}-\right. \\
& \left.-\frac{n\left(\beta E_{\sigma}(k)\right)-n\left(\beta \xi_{|\mathbf{k}-\mathbf{p}|}\right)}{E_{\sigma}(k)-\xi_{|\mathbf{k}-\mathbf{p}|}+i \omega_{n}}\right\}+\ldots
\end{aligned}
$$

де крапками позначено вираз (30) з тією відмінністю, що суми за $\mathbf{k}$ обмежені знизу $k \geq k_{f}$. Якщо тепер розглянути границю $k_{\sigma} / k_{0} \rightarrow 0$ i врахувати, що $k_{f}$ теж мале, то, як не важко бачити, перший доданок формули (31) дає незначний внесок у масовий оператор. 3 іншого боку, враховуючи властивості функцій $I(\omega, k), R(\omega, k)$, практично весь внесок в інтеграл за $\omega$ у формулі (30) йде від околу двох точок $\beta \omega \sim \pm k^{2} / k_{0}^{2}$. Спробуємо "вхопити" цю область трюком, подібним до того, що ми використовували для розрахунку структурного фактора. Будемо вважати, що вираз $(31)$, якщо в ньому замінити $E_{\sigma}(k)$ на $\omega_{\sigma}(k)$ та зняти обмеження в сумі за хвильовим вектором і відкинути доданки, позначені крапками, коректно описує масовий оператор

$$
\Sigma\left(\omega_{n}, p\right) \rightarrow \frac{1}{V} \sum_{\mathbf{k}} \nu_{\sigma}(k) n\left(\beta \xi_{|\mathbf{k}-\mathbf{p}|}\right)-
$$

$$
\begin{aligned}
& -\frac{1}{V} \sum_{\mathbf{k}} \nu_{\sigma}(k) \frac{\rho \nu_{\sigma}(k) \varepsilon_{k}}{\omega_{\sigma}(k)}\left\{\frac{1+n\left(\beta \omega_{\sigma}(k)\right)+n\left(\beta \xi_{|\mathbf{k}-\mathbf{p}|}\right)}{\omega_{\sigma}(k)+\xi_{|\mathbf{k}-\mathbf{p}|}-i \omega_{n}}-\right. \\
& \left.-\frac{n\left(\beta \omega_{\sigma}(k)\right)-n\left(\beta \xi_{|\mathbf{k}-\mathbf{p}|}\right)}{\omega_{\sigma}(k)-\xi_{|\mathbf{k}-\mathbf{p}|}+i \omega_{n}}\right\}
\end{aligned}
$$

тут ми вже використали співвідношення (7) для похідної від дійсної частини поляризаційного оператора. Таким чином, подібно до розрахунку структурного фактора, ми ефективно враховуємо внесок ідеального газу. Щодо виправданості такого трюку при розрахунках, то нижче показано, що отриманий таким чином зсув критичної температури нашої моделі у двох граничних випадках зарядженого бозе-газу високої густини та моделі слабонеідеального газу в головному наближенні повністю збігається із відомими результатами $[2,14]$. Разом з тим, за допомогою виразу (32) можна аналізувати і границю великих значень параметра неідеальності.

\section{4. Границя малих значень параметра неідеальності}

Отже, працюємо далі з виразом (32). Враховуючи те, що в границі $k_{\sigma} / k_{0} \rightarrow 0$ головний внесок в інтеграл за хвильовим вектором будуть вносити малі $k$, то можна замінити бозе-розподіл $n(x)$ на $1 / x$. Тоді, після простого перегрупування доданків з урахуванням рівності (10) для дійсної частини власноенергетичної частини, отримуємо

$$
\begin{aligned}
& \Sigma_{R}(\omega, p)=\frac{1}{\beta V} \sum_{\mathbf{k}} \nu_{\sigma}(k) \frac{\omega_{\sigma}^{2}(k)-2 \rho \nu_{\sigma}(k) \varepsilon_{k}}{\omega_{\sigma}^{2}(k)} \frac{1}{\varepsilon_{|\mathbf{k}-\mathbf{p}|}}- \\
& -\frac{1}{\beta V} \sum_{\mathbf{k}} \nu_{\sigma}(k) \frac{\rho \nu_{\sigma}(k) \varepsilon_{k}}{\omega_{\sigma}^{2}(k)} \frac{\omega}{\varepsilon_{|\mathbf{k}-\mathbf{p}|}}\left\{\frac{1}{\omega_{\sigma}(k)+\varepsilon_{|\mathbf{k}-\mathbf{p}|}-\omega}-\right. \\
& \left.-\frac{1}{\omega_{\sigma}(k)-\varepsilon_{|\mathbf{k}-\mathbf{p}|}+\omega}\right\}
\end{aligned}
$$

Далі, працюючи у межах звичайної теорії збурень, наближено записуємо для перенормованого спектра:

$\widetilde{\varepsilon}_{p}=\varepsilon_{p}+\Sigma_{R}\left(\varepsilon_{p}, p\right)-\Sigma_{R}(0,0)$,

де вже врахували рівність нулю перенормованого хімічного потенціалу $\widetilde{\mu}$. Подальший розрахунок не $\epsilon$ 
складним. Достатньо підставити у формулу (33) тільки довгохвильову асимптотику "спектра":

$\omega_{\sigma}^{2}(k)=2 \rho \nu_{\sigma}(k) \varepsilon_{k}+\frac{2 \zeta(3 / 2)}{\pi^{3 / 2}} \frac{k^{3}}{k_{0}^{3}} T^{2}$.

Легко показати, що головний внесок у величину

$\beta\left[\Sigma_{R}\left(\varepsilon_{p}, p\right)-\Sigma_{R}(0,0)\right] \equiv \sigma\left(p / p_{0}\right)=$

$=\left(2 a_{\sigma} / \pi\right)^{2} I_{\sigma}(\gamma)+\ldots$,

$I_{\sigma}(\gamma)=\int_{0}^{\infty} \frac{d k k}{k^{3-\sigma}+1}\left\{\frac{k}{2 \gamma} \ln \left|\frac{k+\gamma}{k-\gamma}\right|-1\right\}$

де введено такі позначення:

$a_{\sigma}^{3-\sigma}=\pi^{3 / 2}\left(k_{\sigma} / k_{0}\right)^{2-\sigma} / \zeta(3 / 2), \quad \gamma=p /\left(p_{0} a_{\sigma}\right)$.

Тут крапками позначено всі вищі члени розкладу за степенями параметра $a_{\sigma}$. Легко бачити, що інтеграл $I_{\sigma}(\gamma)=-\gamma^{2} \ln (\gamma) / 3$ неаналітичний при малих значеннях аргумента, і форма цієї неаналітичності не залежить від показника степеня $\sigma$ потенціалу взаємодії. Це натяк на універсальну степеневу поведінку $\widetilde{\varepsilon}_{p} \sim p^{2-\eta}$ провідної асимптотики одночастинкового спектра взаємодіючої системи. Важливо, що решта доданків, які не враховані у (36), аналітичні за $\gamma$. Головний внесок у хімічний потенціал системи у критичній точці такий:

$\mu / T_{c}=\frac{\pi}{3-\sigma} \frac{\left(2 a_{\sigma} / \pi\right)^{2}}{\sin (\pi /(3-\sigma))}, \quad \sigma<1$,

де розбіжність при $\sigma>1 \in$ фіктивною і пов'язана із заміною $n(x) \rightarrow 1 / x$ при переході від формули (32) до (33). Щоб знайти критичну температуру нашої моделі, потрібно ще розрахувати величину $Z(p)$ за формулою (27). 3 цією метою обчислюємо похідну

$$
\begin{aligned}
& {\left.\left[\partial \Sigma_{R}(\omega, p) / \partial \omega\right]\right|_{\omega \rightarrow \varepsilon_{p}} \equiv z\left(p / p_{0}\right)=} \\
& =\frac{a_{\sigma}}{\sqrt{\pi} \zeta(3 / 2)} J_{\sigma}(\gamma)+\ldots, \\
& J_{\sigma}(\gamma)=\frac{1}{\gamma} \int_{0}^{\infty} \frac{d k k}{\left(k^{3-\sigma}+1\right)^{2}} \ln \left|\frac{k+\gamma}{k-\gamma}\right|,
\end{aligned}
$$

знову ж таки тут виписаний лише провідний член розкладу за $a_{\sigma}$. Інтеграл $J_{\sigma}(\gamma)$, на відміну від $I_{\sigma}(\gamma)$, аналітичний за $\gamma$ і є константою при малих значеннях аргумента, а на безмежності виходить на асимптотикy $1 / \gamma^{2}$.

Тепер можна взятись за інтегрування формули (28) для рівноважної густини. Виділивши окремо внесок для густини ідеального газу $\rho_{0}, 3$ потрібною нам точністю записуємо

$\rho=\rho_{0}+\frac{p_{0}^{3}}{2 \pi^{2}} \int_{0}^{\infty} d p p^{2}\left\{n\left(p^{2}+\sigma(p)\right)-n\left(p^{2}\right)\right\}+$

$+\frac{p_{0}^{3}}{2 \pi^{2}} \int_{0}^{\infty} d p p^{2} n\left(p^{2}+\sigma(p)\right) z(p)$

після заміни змінної інтегрування $p=a_{\sigma} \gamma$ отримуємо

$\Delta \rho \equiv \rho-\rho_{0}=\frac{p_{0}^{3}}{2 \pi^{2}} a_{\sigma}^{3} \times$

$\times \int_{0}^{\infty} d \gamma \gamma^{2}\left\{n\left(a_{\sigma}^{2} \gamma^{2}+\left(2 a_{\sigma} / \pi\right)^{2} I_{\sigma}(\gamma)\right)-n\left(a_{\sigma}^{2} \gamma^{2}\right)\right\}+$

$+\frac{p_{0}^{3}}{2 \pi^{2}} a_{\sigma}^{3} \int_{0}^{\infty} d \gamma \gamma^{2} n\left(a_{\sigma}^{2} \gamma^{2}+\left(2 a_{\sigma} / \pi\right)^{2} I_{\sigma}(\gamma)\right) \times$

$\times \frac{a_{\sigma}}{\sqrt{\pi} \zeta(3 / 2)} J_{\sigma}(\gamma)$

"витягуючи" головні внески при малих $a_{\sigma}$ :

$\Delta \rho=-\frac{p_{0}^{3}}{2 \pi^{2}} a_{\sigma} \int_{0}^{\infty} d \gamma \frac{(2 / \pi)^{2} I_{\sigma}(\gamma)}{\gamma^{2}+(2 / \pi)^{2} I_{\sigma}(\gamma)}$.

Немає сенсу враховувати вищі степені за параметром $a_{\sigma}$, оскільки ми працюємо в наближенні хаотичних фаз, а пост-RPA внески квадратичні за цим параметром.

Для розрахунку критичної температури $T_{c}$ в цій границі взаємодій використаємо міркування авторів статті [14], де розглядалась бозе-система з точковою взаємодією. Варто відзначити, що RPA не зовсім коректне для моделі газу 3 короткодійними силами. 


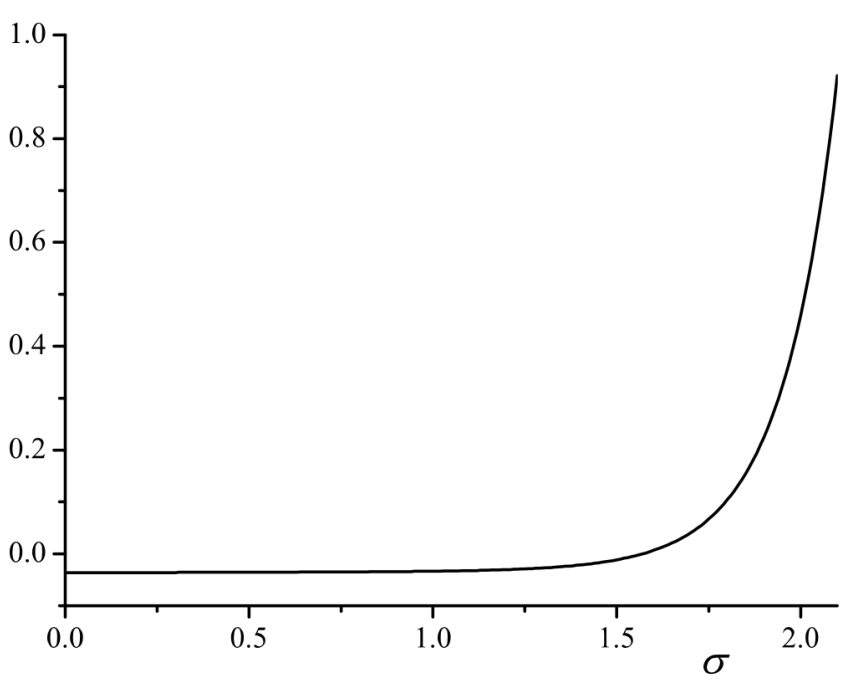

Залежність інтеграла у формулі (39) від показника степеня потенціальної енергї попарної взаємодії між частинками. Мінімальне значення $-0,036$ досягається у кулонівській границі $(\sigma=0)$ і 0,451 для моделі слабонеідеального газу $(\sigma=2)$

Врахуємо, що рівноважна густина не залежить від взаємодії, тоді

$\frac{d}{d a_{\sigma}} \rho=\frac{\partial \rho}{\partial a_{\sigma}}+\frac{\partial \rho}{\partial T_{c}} \frac{\partial T_{c}}{\partial a_{\sigma}}=0$

i, замінюючи з потрібною нам точністю, отримуємо

$\frac{\partial \rho}{\partial T_{c}} \simeq \frac{\partial \rho_{0}}{\partial T_{0}}=\frac{3}{2} \frac{\rho_{0}}{T_{0}}$

або

$\frac{T_{c}(\sigma)-T_{0}}{T_{0}}=-\frac{2}{3} \frac{\Delta \rho}{\rho}$.

Тепер коротко зупинимось на граничних випадках. Спочатку розглянемо модель зарядженого бозе-газу в компенсуючому полі. У цьому випадку величину $a_{0}=\left[\pi^{5 / 2} / 6 \zeta(3 / 2)\right]^{1 / 9} r_{s}^{1 / 3}$ можна переписати через параметр Бракнера. Числовий розрахунок інтеграла (39) для величини $\Delta \rho$ при $\sigma=0$ з урахуванням рівності для зсуву критичної температури дає значення

$\frac{T_{c}(0)-T_{0}}{T_{0}}=-0,026 r_{s}^{1 / 3}$.

Це точний результат для границі великих густин, вперше отриманий іншим методом у роботі [2]. Цікаво, що значення критичної температури зарядженого бозе-газу нижче температури бозе-конденсації ідеального газу.
Друге граничне значення $\sigma=2$ - модель слабонеідеального бозе-газу. Тут $a_{2}=2 \pi^{3 / 2} \rho^{1 / 3} a /[\zeta(3 / 2)]^{1 / 3}$ і зручно записати функцію $I_{2}(\gamma)$ так:

$I_{2}(\gamma)=-\frac{1}{\gamma} \int_{0}^{\gamma} \frac{d y y^{2}}{1-y^{2}} \ln |y|$,

тоді під інтегралом у формулі (39), формально розкладаючи в ряд за $I_{2}(\gamma)$ і обмежуючись першим членом, отримуємо

$\Delta \rho=2 p_{0}^{3} a_{2} \int_{0}^{\infty} \frac{d \gamma}{\gamma^{3}} \int_{0}^{\gamma} \frac{d y y^{2}}{1-y^{2}} \ln |y|$,

тепер, замінивши послідовність інтегрування

$\Delta \rho=2 p_{0}^{3} a_{2} \int_{0}^{1} d y y \int_{0}^{\infty} \frac{d \gamma}{1-\gamma^{2}} \ln |\gamma|$

і підставляючи у вираз для зсуву критичної температури, остаточно одержуємо [14]:

$\frac{T_{c}(2)-T_{0}}{T_{0}}=\frac{8 \pi}{3[\zeta(3 / 2)]^{4 / 3}} \rho^{1 / 3} a=2,328 \rho^{1 / 3} a$.

3 урахуванням числового розрахунку інтеграла (39)

$\frac{T_{c}(2)-T_{0}}{T_{0}}=2,010 \rho^{1 / 3} a$,

що краще наближається до значення МонтеКарло симуляцій $(1,29 \pm 0,05) \rho^{1 / 3} a \quad[15] \quad$ i $(1,32 \pm 0,02) \rho^{1 / 3} a[16]$, та ренормгрупових розрахунків $(1,27 \pm 0,11) \rho^{1 / 3} a$ [17] в семипетльовому наближенні.

\section{5. Висновки}

Працюючи в наближенні хаотичних фаз і використовуючи запропонований тут метод розрахунку власноенергетичної частини одночастинкової функції Гріна, нам вдалось знайти головну поправку для критичної температури моделі бозе-газу з далекодійним відштовхувальним потенціалом $1 / r^{1+\sigma}$ високої густини для випадку $\sigma<1$ і низької густини для випадку $\sigma>1$. Тут важливо наголосити, що RPA $\epsilon$ строгим наближенням у границі малих значень параметра неідеальності тільки для випадку $\sigma<2$. Цікаво, що при $\sigma=1,57$ знак поправки до критичної температури змінюється з від'ємного на додатний. Оскільки експериментальна перевірка, швидше 
за все, неможлива, то було б цікаво порівняти наші результати з розрахунками за допомогою комп'ютерних симуляцій для цієї моделі. Метод обчислення параметрів спектра, запропонований у цій статті, можна узагальнити і на довільну вимірність простору $d>2$, де поведінка системи якісно не буде відрізнятись від розглянутої. У випадку $d \leq 2$ відсутній перехід у стан з бозе-конденсатом при скінченних температурах, i, як наслідок, повністю змінюються властивості моделі. Аналіз спектра колективних збуджень системи вище температури бозеконденсації показав цікаву особливість - для довільного значення параметра неідеальності спектр моделі має точку закінчення. Нагадаємо, що в цьому ж наближенні цієї особливості спектра немає в конденсатній фазі, де поляризаційний оператор (3) має явно полюсний вигляд. У роботі, використовуючи точне співвідношення для першого моменту динамічного структурного фактора, вдалось отримати довгохвильову асимптотику структурного фактора системи.

У майбутньому ми плануємо продовжити дослідження цієї задачі, зокрема, для знаходження критичної температури для всіх значень $k_{\sigma} / k_{0}$, що, очевидно, як і коректний розрахунок наступного члена розкладу за $a_{\sigma}$ у формулі (39), потребує виходу за межі RPA. Також буде розглянуто можливість виникнення нестійкості типу вігнерівської кристалізації в зарядженому бозе-газі вище температури конденсації.

Автор висловлює подяку професорові I.О. Вакарчуку за обговорення результатів роботи, а також О. Менчишину за постійну допомогу.

\section{ДОДАТОК}

Після аналітичного продовження у верхню півплощину дійсна та уявна частини поляризаційного оператора (3) такі:

$\left.\operatorname{Re} \Pi\left(\omega_{n}, k\right)\right|_{i \omega_{n} \rightarrow \omega+i 0}=R(\omega, k)$,

$\left.\operatorname{Im} \Pi\left(\omega_{n}, k\right)\right|_{i \omega_{n} \rightarrow \omega+i 0}=I(\omega, k)$,

де функція

$I(\omega, k)=\frac{1}{16 \pi} \beta k_{0}^{3} \frac{k_{0}}{k} \ln \left|\frac{1-\exp \left\{\beta \mu-\left[k / 2 k_{0}+\beta \omega /\left(2 k / k_{0}\right)\right]^{2}\right\}}{1-\exp \left\{\beta \mu-\left[k / 2 k_{0}-\beta \omega /\left(2 k / k_{0}\right)\right]^{2}\right\}}\right|$,

а дійсну частину можна записати так:

$R(\omega, k)=\beta \rho \frac{k_{0}}{2 k}\left\{f\left(k / 2 k_{0}+\beta \omega /\left(2 k / k_{0}\right), \beta \mu\right)+\right.$

$\left.+f\left(k / 2 k_{0}-\beta \omega /\left(2 k / k_{0}\right), \beta \mu\right)\right\}$.

Тут функція

$f(\varepsilon, y)=\varepsilon \int_{0}^{1} \frac{d x}{\sqrt{1-x}} g_{1 / 2}\left(e^{y-x \varepsilon^{2}}\right) / g_{3 / 2}\left(e^{y}\right)$, $g_{l}\left(e^{y}\right)=\sum_{n>1} \frac{e^{y n}}{n^{l}}$

3 асимптотиками в граничних випадках

$f(\varepsilon \rightarrow 0, y)=2 \varepsilon \frac{g_{1 / 2}\left(e^{y}\right)}{g_{3 / 2}\left(e^{y}\right)}$,

$f(\varepsilon \rightarrow \infty, y)=\frac{1}{\varepsilon}\left\{1+\frac{1}{2 \varepsilon^{2}} \frac{g_{5 / 2}\left(e^{y}\right)}{g_{3 / 2}\left(e^{y}\right)}+\ldots\right\}$,

$f(\varepsilon \rightarrow 0,0)=\left[\pi^{3 / 2} \operatorname{sign}(\varepsilon)+2 \varepsilon \zeta(1 / 2)\right] / \zeta(3 / 2)$,

де $\zeta(x)=\sum_{n \geq 1} 1 / n^{x}$ - дзета функція.

1. L.L. Foldy, Phys. Rev. 124, 649 (1961); 125, 2208 (1962).

2. A.L. Fetter, Ann. Phys. 64, 1 (1971).

3. S. Ma, Phys. Rev. Lett. 29, 1311 (1972).

4. P.V. Panat, H.E. DeWitt, and J.C. Garrison, Phys. Rev. A 8, 3154 (1973).

5. R.F. Bishop, J. Low Temp. Phys. 15, 601 (1974).

6. E.B. Kolomeisky and J.P. Straley , Phys. Rev. B 46, 13942 (1992).

7. T.D. Lee and C.N. Yang, Phys. Rev. 105, 1119 (1957); T.D. Lee, K. Huang, C.N. Yang, Phys. Rev. 106, 1135 (1957).

8. І.О. Вакарчук, В.С. Пастухов, подано до Журн. Фіз. Досл.

9. I.О. Вакарчук, Вступ до проблеми багатъох тіл (Львів, ЛНУ, 1999).

10. S.R. Hore and N.E. Frankel, Phys. Rev. B 12, 2619 (1975).

11. A.L. Fetter and J.D. Walecka, Quantum Theory of ManyParticle Systems (McGraw-Hill, New York, 1971).

12. Д.Н. Зубарев, Усп. физ. наук, 71, 71 (1960).

13. В.С. Пастухов, УФЖ 56, 556 (2011).

14. G. Baym, J.-P. Blaizot, and J. Zinn-Justin, Europhys. Lett. 49, 150 (2000).

15. V.A. Kashurnikov, N.V. Prokof'ev, and B.V. Svistunov, Phys. Rev. Lett. 87, 120402 (2001).

16. P. Arnold and G. Moore, Phys. Rev. Lett. 87, 120401 (2001).

17. B. Kastening, Phys. Rev. A 69, 043613 (2004).

Одержано 09.06.11 


\section{РАСЧЕТ КРИТИЧЕСКОЙ ТЕМПЕРАТУРЫ БОЗЕ-ГАЗА} С ДАЛЬНОДЕЙСТВУЮЩИМИ СИЛАМИ

\section{B.C. Пастухов}

Р е $з$ ю м

В статье проведен расчет критической температуры модели бозе-газа с степенным законом зависимости потенциальной энергии парного взаимодействия от расстояния между частицами. Результат в предельных случаях воспроизводит известные из литературы. В приближении хаотических фаз (RPA) проанализированы параметры спектра коллективных возбуждений модели и получена длинноволновая асимптотика структурного фактора системы выше температуры фазового перехода.

\section{ALCULATION OF CRITICAL TEMPERATURE FOR A BOSE GAS WITH LONG-RANGE FORCES}

\section{V.S. Pastukhov}

Ivan Franko National University of Lviv

(12, Drahomanov Str., Lviv 7900, Ukraine;

e-mail: volodyapastukhov@gmail.com)

\section{$\mathrm{S} \mathrm{u} \mathrm{m} \mathrm{m} \mathrm{a} \mathrm{r} \mathrm{y}$}

The critical temperature has been calculated for a Bose gas with the power-law dependence of the potential energy of interaction between particles on the interparticle distance. The result obtained satisfies the limiting cases known from the literature. The parameters of the collective excitation spectrum of the model have been analyzed in the random phase approximation (RPA). The longwavelength asymptote has been derived for the structure factor of the system above the phase transition temperature. 